\title{
Análisis bibliométrico de las tesis de pregrado en la Facultad de Educación y Ciencias Sociales de la Universidad Adventista de Chile, en el período 2008-2018
}

\author{
Bibliometric analysis of undergraduate theses at the Faculty of Education and \\ Social Sciences of the Adventist University of Chile in Period: 2008-2018
}

\author{
Alixon Reyes Rodríguez ${ }^{a}$ ORCID: 0000-0001-9857-0930 \\ Julia Labra ${ }^{b}$ ORCID: 0000-0002-0075-4326 \\ Bertha Méndez ${ }^{c}$ ORCID: 0000-0001-9353-6725 \\ Lorena Gutiérrez-Jeldres ${ }^{d}$ ORCID: 0000-0002-3589-4249 \\ Leonel Federico-Tuccellie ORCID: 0000-0002-5586-5410 \\ Fanny Vezzoli-Lara ${ }^{\mathrm{f}}$ ORCID: 0000-0001-5750-9017
}

Recibido: 16/06/2020 Aprobado: 7/08/2020

\begin{abstract}
Cómo citar: Reyes Rodríguez, A., Labra, J., Méndez, B., Gutiérrez-Jeldres, L., Federico-Tuccelli, L., \& Vezzoli-Lara, F. (2021). Análisis bibliométrico de las tesis de pregrado en la Facultad de Educación y Ciencias Sociales de la Universidad Adventista de Chile, en el período 2008-2018. Ciencia y Educación, 5(1), 75-93. https://doi.org/10.22206/cyed.2021.v5i1.pp75-93
\end{abstract}

\section{Resumen}

El presente trabajo tiene como propósito el análisis bibliométrico de las tesis realizadas en las carreras de la Facultad de Educación y Ciencias Sociales de la Universidad Adventista de Chile entre 2008 y 2018. Los resultados evidencian 379 investigaciones en modalidad y formato tesis. Los años 2008 y 2018 son los más productivos con 40 estudios cada uno y las carreras más productivas son: Pedagogía en Educación General Básica y Pedagogía en Educación Física, con 79 y 57 trabajos respectivamente. Sobre el $80 \%$ de los tutores tiene posgrado y prevalece la metodología cuantitativa en los estudios. A pesar del universo de trabajos finalizados, no se priorizó la publicación de estos en su momento. La investigación en pregrado apunta al enfoque cuantitativo,

\begin{abstract}
The purpose of this work is the bibliometric analysis of the theses carried out in the careers of the Faculty of Education and Social Sciences of the Adventist University of Chile between 2008 and 2018. The results show 379 investigations in thesis format and format. The years 2008 and 2018 are the most productive with 40 studies each and the most productive careers are: Pedagogy in Basic General Education and Pedagogy in Physical Education, with 79 and 57 jobs respectively. Over $80 \%$ of the tutors have a postgraduate degree and the quantitative methodology prevails in the studies. Despite the universe of completed works, the publication of these was not prioritized at the time. Undergraduate research points to the
\end{abstract}

\footnotetext{
a Pedagogía en Educación Física, Universidad Adventista de Chile, Chillán, Chile. Correo-e: alixonreyes@unach.cl

b Pedagogía en Biología y Ciencias Naturales, Universidad Adventista de Chile, Chillán, Chile. Correo-e: julialabra@unach.cl

c Pedagogía en Historia, Universidad Adventista de Chile, Chillán, Chile. Correo-e: berthamendez@unach.cl

d Pedagogía en Educación General Básica, Universidad Adventista de Chile, Chillán, Chile. Correo-e: lorenagutierrez@unach.cl

e Pedagogía en Educación Física, Universidad Adventista de Chile, Chillán, Chile. Correo-e: leonelfederico@alu.unach.cl

${ }^{\text {f }}$ Pedagogía en Inglés, Universidad Adventista de Chile, Chillán, Chile. Correo-e: fannyvezzoli@alu.unach.cl
} 
elemento que implica fomentar otros enfoques para el abordaje y la diversidad epistemológica, más aún al tratarse de una facultad de Educación y Ciencias Sociales.

Palabras clave: bibliometría; investigación; educación; metodología; trabajos finales de carrera.

\section{Introducción}

La investigación es un proceso indispensable en el contexto universitario, a tal punto, que no se entiende la idea de universidad sin la investigación, o sin que esta se convierta en el eje transversal del quehacer en estos espacios, tanto en pregrado como en posgrado (Arellano-Sacramento et al., 2017; Ganga et al., 2019; Moreira et al., 2019). La investigación y la producción científica se asocian cada vez más a parámetros con los que las agencias de acreditación (de los países que cuentan con estos sistemas), intentan medir variables de calidad (Barra, 2019; Piedra y Martínez, 2007). Considerando este contexto, la investigación trata de un proceso que, entre sus virtudes están: la generación y la transferencia de conocimiento (Bird, 2014; Hirsch, 2020), el flujo constante de la renovación docente, la consolidación de espacios formativos, el desarrollo de habilidades investigativas en estudiantes de pregrado y postgrado, la adquisición de habilidades de investigación y búsqueda de información que fortalecen el desempeño laboral, la articulación de los esfuerzos de vinculación de la universidad con el entorno, el desarrollo de la institución, de una región o de un país (Valle y Salvador, 2009). De allí que, fomentar y potenciar la investigación no sea un asunto menor en la formación universitaria.

Históricamente las universidades han optado, incluso desde sus inicios, Bolonia y París (Chuaqui, 2002; Moncada, 2008), por la tesis como un mecanismo con múltiples finalidades (Ibarra-López, 2017). Esto es, como un documento científico-académico a través del cual se informa y argumenta (Alonso, 2008); como un requisito para demostrar la probidad del grado académico respectivo (Arnoux, 2008); como medio de quantitative approach, an element that implies promoting other approaches to the approach and epistemological diversity, especially since it is a faculty of Education and Social Sciences.

Key Words: bibliometry; research; education; methodology; final studies.

difusión y/o como un proceso formativo (Sánchez, 2014). Ahora bien, la tesis también puede ser concebida como una instancia propicia para el desarrollo de habilidades investigativas asociadas a aspectos valóricos, técnicos y profesionales necesarios que apuntan a la optimización del desempeño laboral. Siendo de esta forma, la tesis se ha mantenido como uno de los hitos referentes en la formación universitaria dado su compromiso con la investigación y su impacto social.

La Universidad Adventista de Chile (UnACh), comprendiendo este flujo, ha adoptado como una de sus más importantes premisas:

Promover acciones de investigación y divulgación de las ciencias, artes y cultura, fomentando en los miembros de su comunidad docente-estudiantil una actitud investigadora que tenga en cuenta tanto los datos aportados por la investigación científica y la razón humana, como los entregados al hombre por medio de la Revelación. (UnACh, 2018, p. 14)

Esta idea ha venido siendo potenciada desde las carreras ofrecidas en la institución en las diferentes facultades, y las carreras asociadas a pedagogía y ciencias sociales radicadas en la Facultad de Educación y Ciencias Sociales (FECS), las cuales, responden a este desafío institucional. Una muestra de ello son los perfiles de egreso de las diferentes carreras de la FECS, en los que esta concepción se encuentra vertida, y que desde la malla curricular se plantea como propuesta formativa en las asignaturas destinadas de manera específica, para atender la formación en investigación; esto es, Formulación de Proyectos, Métodos y Técnicas de Investigación, Trabajo Final de Carrera (TFC). Esta línea formativa conduce la formación de habilidades específicas en investigación, que los estudiantes 
de las carreras asociadas a la FECS deben desarrollar y concretar en un trabajo de investigación denominado Trabajo Final de Carrera (TFC) como requisito de titulación. En este sentido, y desde los cierres de cada una de las cohortes en cada una de las carreras, se ha evidenciado la producción de investigación en tales contextos.

Conocer cuál es el aporte que en materia de investigación ha generado la FECS desde estas instancias, y muy especialmente desde los TFC; es el propósito de esta investigación. Para ello se han considerado aspectos que van desde la iniciación de un trabajo de investigación hasta su publicación en revistas científicas, entendiéndose que la divulgación de la investigación se constituye hoy en una necesidad (Castro-Rodríguez, 2018), y más aún cuando se comprende que la investigación en pregrado favorece las posibilidades de seguir investigando durante el desempeño profesional (Huamaní et al., 2008; Reinders et al., 2005). Así pues, el centro de interés del presente trabajo pasa por reconocer, caracterizar y analizar los TFC realizados en la FECS de la UnACh, anteriormente denominados como trabajos finales de integración (TFI), en el período transcurrido entre 2008 y 2018. De acuerdo con el Reglamento del Trabajo Final de Carrera para los Estudios de Pregrado (2017), el TFC se define de la siguiente manera:

El Trabajo Final de Carrera (TFC) es una actividad curricular para la obtención del grado del Licenciado o del Título Profesional de nivel superior, donde un estudiante, o un grupo de estudiantes, realiza un estudio en profundidad en alguna problemática específica de su especialidad y formula, elabora y desarrolla un proyecto de investigación o aplicación o intervención; o colabora en un proyecto de investigación o aplicación o intervención dirigido por un académico de la UnACh o de otro centro de educación superior o de investigación en convenio con la UnACh (Universidad Adventista de Chile, 2017; Art. 1).

Al mismo tiempo el reglamento especifica tres modalidades: tesis, proyecto y colaboración, pudiendo ser desarrollado de forma individual o grupal. El propósito de esta instancia es:
... que el estudiante se inicie en el manejo de las herramientas básicas de investigación o aplicación o intervención con el máximo rigor científico, y ello además le permita articular de forma óptima su proceso de desarrollo como profesional o en una posterior etapa de formación de posgrado (Ídem, Art. 2).

El TFC, en todo caso, da respuesta a la necesidad de formación inicial del estudiantado en materia de investigación, ofreciendo instancias y procesos fundamentales para el desarrollo de habilidades, valores, conocimientos y prácticas específicas, proveyendo herramientas elementales para conocer su entorno profesional de forma sistemática e interactuando, a fin de transformar las realidades susceptibles de cambio, en el contexto pedagógico y disciplinar.

\section{Marco teórico}

El análisis de la producción científica es un ejercicio que ha cobrado mucho interés en los últimos años (Aleixandre-Benavent et al., 2017) dado el aporte que representa para el contexto académico y científico. Este tipo de estudios se realiza partiendo de la cienciometría (Millán et al., 2017), y esta última tiene que ver con el estudio de los aspectos cuantitativos de la ciencia como disciplina (Michán y Muñoz-Velasco, 2013). La bibliometría, que se desprende de la cienciometría, se refiere a la cuantificación de indicadores que permiten medir la producción científica (Licea, Santillán-Rivero, 2002), cobrando cada vez mayor importancia en el contexto académico, dada la alta producción científica a nivel mundial en todos los campos del saber (Madrid et al., 2017), y que puede evidenciarse en diversas modalidades.

Los orígenes de la bibliometría pueden remontarse, según Cortés (2007), quien hace una referencia a Zbikowska-Migon, al siglo XviII; con los estudios realizados por Frömmichen y Balbi, en relación con la producción y el comercio alemán del libro. No obstante, la misma dinámica de la divulgación del conocimiento, el crecimiento de la revista científica y su consolidación como medio predilecto para la divulgación del conocimiento (Reyes y Moraga, 2020), han 
potenciado la bibliometría como campo de estudio para el comportamiento de la literatura científica.

Según Tomás-Górriz y Tomás-Casterá (2018), la bibliometría, juntamente con la cienciometría y la informetría, atienden al análisis y la evaluación de la información, así como el conocimiento resultante de la actividad científica, elemento fundamental para todos los programas relacionados con la investigación pública, la tecnología y el desarrollo social. Así, la bibliometría está asociada a la medición de la literatura científica, especialmente en lo referente a las citas, lo que incluye producciones como artículos científicos, tesis, actas de congresos, libros, entre otros.

En tal sentido, dado que el interés de este trabajo radica en el análisis bibliométrico de las tesis de pregrado de la FECS de la universidad, es importante destacar que este análisis permite conocer los niveles de productividad de esta, en términos de los TFC (o tesis) elaborados por los estudiantes, que a su vez son guiados por profesores de las respectivas carreras. Este ejercicio, además de tener validez metodológica y científica debido a que el análisis corresponde a grandes unidades [TFC de 11 carreras entre 2008 y 2018 (Aleixandre-Benavent et al., 2017)], permite además conocer la tendencia en la producción de conocimiento (productividad, líneas de investigación, temáticas específicas, áreas de formación, metodologías empleadas, divulgación en revistas científicas), y sirve como indicador de logro en cuanto a la tributación de la productividad en relación con el perfil de egreso de los estudiantes que son formados en la universidad. Asimismo, conocer las tendencias en la producción de conocimiento en educación tiene una importancia capital, dado a que se encuentra asociado a la consolidación de una episteme en términos de generación de conocimiento en el campo, en relación con las tendencias de producción a nivel nacional e internacional (Arias y Navarro, 2017).

Para el análisis bibliométrico, se usan ciertos marcadores denominados indicadores bibliométricos. Estos son datos muy específicos que cuantifican elementos clave de la producción científica (Maggiorani et al, 2019; Velasco et al., 2012), y serán esos los datos cuan- tificables que constatan el crecimiento y desarrollo de la investigación, además de que permiten evaluar el comportamiento de la información científica (Peralta, Frías y Chaviano, 2015). En el caso que nos atañe, la producción de literatura científica de la FECS a partir de los TFC, se han encontrado algunos antecedentes que demarcan las lógicas de la investigación bibliométrica en la UnACh.

En la búsqueda de información se ha logrado ubicar diversidad de estudios bibliométricos, entre ellos, algunos realizados en función de la producción científica de revistas y/o de índices de revistas, eventos académicos, programas científicos, de repositorios y bases de datos, proyectos, además de la producción científica de carreras particulares.

El caso que convoca este trabajo, se referencian estudios bibliométricos en facultades de diferentes universidades. Este tipo de estudios pueden incorporar tesis en pregrado y postgrado, proyectos de investigación, artículos en revistas científicas, libros arbitrados, etc. No obstante, en el presente ejercicio nos enfocaremos en aquellos que han sido desarrollados considerando solo las tesis de pregrado e incluyen indicadores bibliométricos específicos como muestra y patrón para este trabajo. Siendo así, se parte en primera instancia con una investigación realizada por Coto (2020) en relación con los proyectos de grado en la Escuela de Ingeniería Eléctrica de la Universidad de Costa Rica, en el período 1999-2018. El propósito de dicho estudio fue realizar un análisis descriptivo de la distribución temporal y temática de los proyectos de grado respectivos, considerando para ello las siguientes variables (o indicadores): número de proyectos por quinquenio, áreas temáticas, páginas por documento y género de los investigadores.

Por otro lado, se referencia a Agramonte et al. (2018), quienes hicieron un análisis bibliométrico de la producción científica de la Facultad de Comunicación de la Universidad de La Habana (Cuba) en relación con las Tecnologías de la Información y Comunicación. También, en este trabajo se analizaron los indicadores asociados a productividad por tutores (cantidad de tesis guiadas por los profesores guías), tutorías en 
colaboración, productividad por departamentos o unidades, productividad por año, temas de investigación, concurrencia de palabras clave.

También, se encontró un estudio desarrollado por Moreira et al. (2019), en el que se realiza un análisis bibliométrico de los trabajos de titulación de la Escuela de Ciencias de la Educación de la Pontificia Universidad Católica del Ecuador, Sede Santo Domingo, considerando el período 2000-2016. En dicho trabajo se emplearon indicadores orientados a identificar las palabras significativas de los títulos; a analizar técnicas e instrumentos, y, finalmente, un indicador de pertinencia, que está dirigido a medir las asignaturas o áreas en la que fueron aplicados dichos trabajos.

Asimismo, se encontró el trabajo de Castro-Rodríguez (2018) en relación con el análisis bibliométrico de la producción científica en la Facultad de Odontología de la Universidad Nacional Mayor de San Marcos (Perú), basado en las tesis de pregrado realizadas entre 2013 y 2017. Los indicadores considerados fueron: índice de productividad, productividad por género, publicación científica (trabajos publicados en revistas científicas), índice de citación (asociado al factor de impacto), diseño metodológico de la investigación, área temática y redes de colaboración.

Muñoz y Suárez (2012), por otro lado, realizaron un análisis bibliométrico de los trabajos de grado en la Facultad de Psicología de la Universidad San Buenaventura de Bogotá, en el período que transcurre entre 2002 y 2010. Allí tomaron en cuenta indicadores temáticos (líneas de investigación, temas de investigación, áreas de la psicología), indicadores metodológicos (tipo de investigación, muestra, edad de los sujetos considerados como unidades muestrales, instrumentos), e indicadores temporales (relación de trabajos y cantidad por año, estudiantes graduados). A su vez, Valle y Salvador (2009) desarrollaron un análisis bibliométrico de las tesis de pregrado de la Facultad de Medicina de la Universidad Nacional Mayor de San Marcos (Perú), en un período que abarcó entre 1998 y 2008, considerando indicadores como: cantidad de tesis, áreas de investigación, especialidades y diseños metodoló- gicos, colaboración entre especialidades, proporción de tesis publicadas en una revista indizada.

San Martín y Pacheco (2008), en su momento, hicieron un análisis bibliométrico de las tesis de pregrado de la Facultad de Medicina Veterinaria de la Universidad Nacional Mayor de San Marcos, entre 2001 y 2006. Los indicadores tomados en cuenta fueron: cantidad de tesis realizadas por áreas, por disciplina y por departamento, directores de tesis. El último trabajo traído a consideración es el de Jiménez (2004), caso que se aproxima a la línea de interés del presente estudio, quien hizo un análisis bibliométrico comparando la producción de las tesis de pregrado de las Escuelas de Educación de la Universidad Central de Venezuela y la Universidad Nacional Experimental Simón Rodríguez, entre los años 1990 y 1999. Los indicadores que usó para el estudio fueron: cantidad de tesis por universidad, por año y por carrera (de las correspondientes escuelas de Educación de cada universidad analizada), palabras clave empleadas en cada tesis, tutores de las tesis, metodología empleada en las tesis, autores más consultados, autores institucionales más citados, revistas más consultadas, referencias electrónicas, etc. Todos estos trabajos emplean indicadores bibliométricos a fin de considerar la productividad científica y la tendencia de investigación en materia de tesis o trabajos finales de carrera desarrollados por estudiantes de pregrado. Así, nos dan la medida de los indicadores bibliométricos que se emplean en este tipo de estudios, entendiéndose que estos sirven también a los propósitos de la facultad que en todo caso oficia la investigación.

Conocer y caracterizar la producción científica de una facultad en términos de las tesis de pregrado puede servir para determinar mejoras y generar procesos decisivos, en aras de optimizar la formación de los estudiantes y profesores; profundizar y proyectar áreas en las que puedan existir falencias, en función de la concentración de esfuerzos en áreas estratégicas, entre otros aspectos. De allí la importancia de trabajos como los ya mencionados y como el que se desarrolla en el ejercicio presente. 
Análisis bibliométrico de las tesis de pregrado en la Facultad de Educación y Ciencias Sociales de la Universidad Adventista de Chile, en el periodo 2008-2018

\section{Metodología}

El presente trabajo sigue una metodología cuantitativa, del tipo analítico, de carácter descriptivo-retrospectivo en torno a la producción de los TFC en la FECS, entre 2008 y 2018 (ambos años inclusive). La unidad de análisis está considerada por los TFC realizados por los estudiantes de pregrado de cada una de las carreras de la FECS, como requisito parcial para la obtención del título profesional y el grado académico correspondiente. La búsqueda de información se realizó en agosto y septiembre de 2019 en el archivo de la Coordinación de los TFC de la FECS, el archivo de la Biblioteca 'Mariano Renedo Lucero' y en el repositorio digital de la universidad (http://bibliorepositorio. unach.cl/). Se procedió a la localización y recuperación de los trabajos inextenso en formato PDF, formato Word y los trabajos impresos archivados.

Posteriormente, se procedió a hacer un recuento manual en directo desde la fuente, avanzando así un proceso de curaduría de datos para optimizar en mayor medida el proceso de análisis. También, siguiendo el modelo orientado por Lagos y Pérez-Gutiérrez (2016), se hizo la descripción bibliográfica o análisis formal de Garrido (2011) y la correspondiente clasificación disciplinar, atendiendo a las normas internacionales de catalogación de la International Organization for Standardization (ISO, 2010). Finalmente, se hizo un rastreo de coincidencias para la relación entre TFC y artículos publicados, en Google Scholar, Lilacs, PubMed, Latindex, Scielo, Scopus y WoS, además de la consulta a los informes de autoevaluación de las carreras de la FECS y la consulta a los directores de carrera correspondientes. Se utilizó el programa Excel 2016 (Hoja de cálculo diseñada por Microsoft Office para Windows).

Para la selección de los indicadores bibliométricos, se han considerado estudios desarrollados en este ámbito que nos permiten establecer patrones metodológicos coherentes con las unidades muestrales (tesis de pregrado) y los objetivos del estudio. Así, los indicadores bibliométricos considerados en el estudio fueron: cantidad de trabajos por año y trabajos totales por carrera, profesores guía por carrera y grados académicos de los mismos, líneas de investigación por carrera, cantidad de TFC por línea, cantidad de TFC según metodología (cuantitativa, cualitativa, mixta), cantidad de TFC según modalidad (tesis, proyecto, colaboración). Como se ha mencionado anteriormente, tales indicadores han sido considerados dadas las evidencias mostradas en trabajos realizados con características y unidades muestrales similares (Jiménez, 2004; Lagos y Pérez-Gutiérrez, 2016).

\section{Resultados}

A continuación, se presentan los resultados obtenidos de los indicadores bibliométricos anteriormente señalados.

Tabla 1.

Relación de carreras FECS y trabajos finales de carrera

\begin{tabular}{|c|c|c|c|c|c|c|c|c|c|c|c|c|}
\hline Carrera & 2008 & 2009 & 2010 & 2011 & 2012 & 2013 & 2014 & 2015 & 2016 & 2017 & 2018 & Totales \\
\hline Educación Física & 0 & 0 & 0 & 8 & 11 & 4 & 7 & 6 & 5 & 7 & 9 & 57 \\
\hline Matemática y Computación & 1 & 2 & 2 & 2 & 1 & 1 & 3 & 1 & 1 & 0 & 1 & 15 \\
\hline Religión & 1 & 0 & 0 & 0 & 1 & 1 & 0 & 0 & 0 & 0 & 0 & 3 \\
\hline Inglés & 3 & 3 & 5 & 4 & 3 & 3 & 2 & 2 & 3 & 2 & 3 & 33 \\
\hline Biología y Ciencias Naturales & 3 & 4 & 2 & 4 & 2 & 1 & 1 & 1 & 1 & 1 & 1 & 21 \\
\hline Música & 4 & 2 & 6 & 3 & 3 & 4 & 4 & 5 & 5 & 8 & 5 & 49 \\
\hline Trabajo Social & 4 & 5 & 4 & 3 & 2 & 5 & 3 & 4 & 2 & 3 & 7 & 42 \\
\hline Educación Parvularia & 1 & 0 & 2 & 2 & 2 & 1 & 5 & 3 & 3 & 5 & 7 & 31 \\
\hline Educación General Básica & 15 & 11 & 10 & 8 & 5 & 7 & 6 & 4 & 5 & 5 & 3 & 79 \\
\hline Historia y Geografía & 3 & 1 & 4 & 3 & 3 & 1 & 3 & 2 & 1 & 0 & 2 & 23 \\
\hline Lengua Castellana y Comunicación & 5 & 3 & 3 & 2 & 3 & 2 & 1 & 2 & 2 & 1 & 2 & 26 \\
\hline Totales & 40 & 31 & 38 & 39 & 36 & 30 & 35 & 30 & 28 & 32 & 40 & 379 \\
\hline
\end{tabular}


Como se observa en la tabla 1, la FECS contabiliza una producción de 379 TFC en total, en el período en estudio, considerando una relación que oscila entre $40 \pm 28$. Las carreras de mayor productividad en función de los TFC entre 2008 y 2018 son las carreras de Pedagogía en Educación General Básica y Pedagogía en Educación Física (estando esto asociado a la cantidad de estudiantes matriculados, siendo estas dos carreras las que han contado con mayor cantidad de estudiantes en la FECS, seguidas por Pedagogía en Música y Trabajo Social). Los años 2008 y 2011 repuntan como los años más productivos en términos genéricos de la FECS.

Tabla 2.

Profesores guía TFC y grados académicos

\begin{tabular}{|c|c|c|c|c|}
\hline Carrera & $\begin{array}{c}\text { Título } \\
\text { pregrado }\end{array}$ & Maestría & Doctorado & Totales \\
\hline $\begin{array}{l}\text { Educación } \\
\text { Física }\end{array}$ & 3 & 8 & 3 & 14 \\
\hline $\begin{array}{l}\text { Matemática y } \\
\text { Computación }\end{array}$ & 1 & 5 & 1 & 7 \\
\hline Religión & 0 & 1 & 0 & 1 \\
\hline Inglés & 3 & 10 & 0 & 13 \\
\hline $\begin{array}{l}\text { Biología y } \\
\text { Ciencias } \\
\text { Naturales }\end{array}$ & 2 & 7 & 3 & 12 \\
\hline Música & 4 & 6 & 3 & 13 \\
\hline Trabajo Social & 1 & 4 & 0 & 5 \\
\hline $\begin{array}{l}\text { Educación } \\
\text { Parvularia }\end{array}$ & 1 & 8 & 0 & 9 \\
\hline $\begin{array}{c}\text { Educación } \\
\text { General Básica }\end{array}$ & 3 & 18 & 3 & 24 \\
\hline $\begin{array}{l}\text { Historia y } \\
\text { Geografía }\end{array}$ & 1 & 7 & 3 & 11 \\
\hline $\begin{array}{c}\text { Lengua } \\
\text { Castellana y } \\
\text { Comunicación }\end{array}$ & 1 & 8 & 0 & 9 \\
\hline Totales & $\begin{array}{c}20 \\
(16.95 \%)\end{array}$ & $\begin{array}{c}82 \\
(69.49 \%)\end{array}$ & $\begin{array}{c}16 \\
(13.56 \%)\end{array}$ & $\begin{array}{c}118 \\
(100 \%)\end{array}$ \\
\hline
\end{tabular}

La carrera que más ha empleado a docentes en el trabajo de asesoría de los TFC ha sido la carrera de Pedagogía en Educación General Básica, con 24 docentes, seguido de la carrera de Pedagogía en Educación Física con 14, y luego con 13, las carreras de Pedagogía en Inglés y Pedagogía en Música. La mayoría de los docentes en guía de TFC poseen el grado académico de Magíster (69.49\%), el 13.56 \% (16) de docentes tienen doctorado y el 16,95\% restante cuenta con título de pregrado. Esto es, el 83.05\% de los docentes que han conducido TFC en el período en estudio, son docentes con estudios de posgrado.

Tabla 3.

Profesores con mayor cantidad de TFC por carrera

\begin{tabular}{|l|c|c|}
\hline \multicolumn{1}{|c|}{ Carrera } & Profesor & Cantidad tfc \\
\hline Educación Física & Rodrigo Moraga & 15 \\
\hline $\begin{array}{l}\text { Matemática y } \\
\text { Computación }\end{array}$ & Anny Acuña & 5 \\
\hline Religión & Alexis Fernández & 8 \\
\hline Inglés & Alejandro Marchant & 7 \\
\hline $\begin{array}{l}\text { Biología y Ciencias } \\
\text { Naturales }\end{array}$ & Rodrigo Quezada & 13 \\
\hline Música & Dan Belmar & 20 \\
\hline Trabajo Social & Bernarda Muñoz & 10 \\
\hline Educación Parvularia & Chery Leiva & 18 \\
\hline Educación General Básica & Marcos Parada & 7 \\
\hline Historia y Geografía & César Ojeda & 10 \\
\hline $\begin{array}{l}\text { Lengua Castellana y } \\
\text { Comunicación }\end{array}$ & & 3 \\
\hline
\end{tabular}

Los docentes con mayor cantidad de TFC por carrera se presentan, varios de ellos con más de 10 TFC conducidos (tabla 3). Sin embargo, vale destacar que, los más productivos en estos términos en razón de toda la FECS (tabla 4), vendrían a ser Chery Leiva con 21 TFC [Pedagogía en Religión (carrera desincorporada de la oferta curricular) y Pedagogía en Educación General Básica] y Dan Belmar con 20 TFC de Trabajo Social. 
Análisis bibliométrico de las tesis de pregrado en la Facultad de Educación y Ciencias Sociales de la Universidad Adventista de Chile, en el periodo 2008-2018

Tabla 4.

Profesores con mayor cantidad de TFC en FECS

\begin{tabular}{|l|c|c|}
\hline \multicolumn{1}{|c|}{ Profesor(a) } & $\begin{array}{c}\text { Cantidad } \\
\text { TFC }\end{array}$ & Carreras \\
\hline Chery Leiva & 21 & $\begin{array}{c}\text { Pedagogía en Religión; Pedagogía } \\
\text { en Educ. General Básica }\end{array}$ \\
\hline Dan Belmar & 20 & Trabajo Social \\
\hline Massiel Venegas & 18 & Trabajo Social \\
\hline Rodrigo Moraga & 15 & Pedagogía en Educación Física \\
\hline $\begin{array}{l}\text { Isidro Sazo } \\
\text { Rodrigo } \\
\text { Quezada }\end{array}$ & 14 & $\begin{array}{c}\text { Pedagogía en Educación General } \\
\text { Básica }\end{array}$ \\
\hline $\begin{array}{l}\text { Bernarda } \\
\text { Muñoz }\end{array}$ & 10 & Pedagogía en Educación Parvularia \\
\hline César Ojeda & 10 & $\begin{array}{c}\text { Pedagogía en Lenguaje y } \\
\text { Comunicación }\end{array}$ \\
\hline
\end{tabular}

\section{Tabla 5.}

TFC según lineas de investigación por carrera FECS

\begin{tabular}{|l|l|c|}
\hline \multicolumn{1}{|c|}{ Línea de Investigación } & \multicolumn{1}{|c|}{ Carrera } & F \\
\hline $\begin{array}{l}\text { Otros Pedagogía Educación } \\
\text { General Básica }\end{array}$ & $\begin{array}{l}\text { P. Educación General } \\
\text { Básica }\end{array}$ & 30 \\
\hline $\begin{array}{l}\text { Actividad Física, Ejercicio Físico y } \\
\text { Salud }\end{array}$ & P. Educación Física & 25 \\
\hline Didáctica de la enseńanza & $\begin{array}{l}\text { P. Educación General } \\
\text { Básica }\end{array}$ & 25 \\
\hline $\begin{array}{l}\text { Metodologías y evaluación en } \\
\text { Educación Parvularia }\end{array}$ & $\begin{array}{l}\text { P. Educación } \\
\text { Parvularia }\end{array}$ & 24 \\
\hline $\begin{array}{l}\text { Formación ciudadana, valores e } \\
\text { interculturalidad }\end{array}$ & $\begin{array}{l}\text { P. Educación General } \\
\text { Básica }\end{array}$ & 17 \\
\hline $\begin{array}{l}\text { Metodología y didáctica de la } \\
\text { enseńanza del inglés }\end{array}$ & P. Inglés & 16 \\
\hline Grupos Sociales & Trabajo Social & 15 \\
\hline $\begin{array}{l}\text { Modelos de enseñanza en } \\
\text { Educación Física y Deporte }\end{array}$ & P. Educación Física & 13 \\
\hline Familia y relaciones de género & Trabajo Social & 12 \\
\hline Folclore y tradiciones & P. Música & 12 \\
\hline Literatura inglesa & P. Inglés & 11 \\
\hline Música y aprendizaje & P. Música \\
\hline Historia y geografía regional & P. Historia y Geografía & 10 \\
\hline Literatura & $\begin{array}{l}\text { P. Lengua Castellana y } \\
\text { Comunicación }\end{array}$ & 10 \\
\hline Educación & $\begin{array}{l}\text { P. Lengua Castellana y } \\
\text { Comunicación }\end{array}$ & 10 \\
\hline
\end{tabular}

\begin{tabular}{|c|c|c|}
\hline $\begin{array}{l}\text { Motricidad Humana: Evaluación, } \\
\text { Prescripción y Rendimiento }\end{array}$ & P. Educación Física & 10 \\
\hline Otros Música & P. Música & 10 \\
\hline Educación Física Inclusiva & P. Educación Física & 9 \\
\hline Didáctica de las ciencias & $\begin{array}{l}\text { P. Biología y Ciencias } \\
\text { Naturales }\end{array}$ & 9 \\
\hline Didáctica educacional & P. Historia y Geografía & 8 \\
\hline $\begin{array}{l}\text { Didáctica y conceptualización } \\
\text { matemática }\end{array}$ & $\begin{array}{l}\text { P. Matemática y } \\
\text { Computación }\end{array}$ & 8 \\
\hline $\begin{array}{l}\text { Familia y comunidad: su } \\
\text { integración al proceso educativo }\end{array}$ & $\begin{array}{l}\text { P. Educación } \\
\text { Parvularia }\end{array}$ & 7 \\
\hline Formación inicial docente & $\begin{array}{l}\text { P. Educación General } \\
\text { Básica }\end{array}$ & 7 \\
\hline Educación extraescolar & P. Música & 7 \\
\hline Música y Salud & P. Música & 6 \\
\hline Medio Ambiente & $\begin{array}{l}\text { P. Biología y Ciencias } \\
\text { Naturales }\end{array}$ & 6 \\
\hline Otros Trabajo Social & Trabajo Social & 6 \\
\hline Historia denominacional & P. Historia y Geografía & 5 \\
\hline Lingüística aplicada & P. Inglés & 5 \\
\hline Otros Biología y Ciencias Naturales & $\begin{array}{l}\text { P. Biología y Ciencias } \\
\text { Naturales }\end{array}$ & 5 \\
\hline $\begin{array}{l}\text { Desarrollo, gestión y planeación } \\
\text { participativa }\end{array}$ & Trabajo Social & 5 \\
\hline Enfoque social de la salud & Trabajo Social & 4 \\
\hline Lingüística & $\begin{array}{l}\text { P. Lengua Castellana y } \\
\text { Comunicación }\end{array}$ & 4 \\
\hline $\begin{array}{l}\text { Desarrollo del pensamiento } \\
\text { matemático }\end{array}$ & $\begin{array}{l}\text { P. Matemática y } \\
\text { Computación }\end{array}$ & 4 \\
\hline Religión & P. Religión & 3 \\
\hline $\begin{array}{l}\text { Realidad educativa a nivel nacional } \\
\text { en el contexto de la educación }\end{array}$ & $\begin{array}{l}\text { P. Matemática y } \\
\text { Computación }\end{array}$ & 3 \\
\hline $\begin{array}{l}\text { Actualidad y música } \\
\text { contemporánea }\end{array}$ & P. Música & 2 \\
\hline Comunicación & $\begin{array}{l}\text { P. Lengua Castellana y } \\
\text { Comunicación }\end{array}$ & 2 \\
\hline Psicolingüística & P. Inglés & 1 \\
\hline Formación inicial docente & $\begin{array}{l}\text { P. Biología y Ciencias } \\
\text { Naturales }\end{array}$ & 1 \\
\hline Creación y composición musical & P. Música & 1 \\
\hline $\begin{array}{l}\text { Lectura de textos y argumentación } \\
\text { científica }\end{array}$ & $\begin{array}{l}\text { P. Biología y Ciencias } \\
\text { Naturales }\end{array}$ & 0 \\
\hline Pensamiento científico & $\begin{array}{l}\text { P. Biología y Ciencias } \\
\text { Naturales }\end{array}$ & 0 \\
\hline $\begin{array}{l}\text { Conceptualización y aprendizaje de } \\
\text { la matemática }\end{array}$ & $\begin{array}{l}\text { Matemática y } \\
\text { Computación }\end{array}$ & 0 \\
\hline
\end{tabular}


Entre todas las carreras de la FECS hay declaradas 40 líneas de investigación, no obstante, hay TFC que por la temática desarrollada no corresponden a ninguna de las líneas declaradas por las carreras en cuestión (Educación General Básica, Trabajo Social, Música, Biología y Ciencias Naturales), de allí que esos TFC se hayan agrupado en una categorización denominada OTROS, agregando el nombre de la carrera respectiva (tabla 5). Así, tendríamos finalmente 40 líneas definidas y cuatro grupos de TFC a ser caracterizados, según nuevas creaciones y/o redefinición de las líneas existentes.

Hay líneas que han sido muy productivas en función de los TFC, y en ese grupo se encuentran 17 líneas de investigación que tienen 10 o más TFC (estando en ese grupo, dos de los cuatro grupos que no son caracterizados en líneas específicas); cuatro de las líneas tienen más de 20 TFC (estando en este grupo, uno de los cuatro grupos que no son caracterizados en líneas específicas, correspondiente a Educación General Básica, que de hecho, es el que mayor producción registra, con 30 TFC). Si sacamos el grupo de TFC con mayor producción caracterizado como "Otros", de Educación General Básica, tendríamos que las líneas de investigación más productivas son las de "Actividad física, ejercicio físico y salud” (Pedagogía en Educación Física), y "Didáctica de la enseñanza" (Pedagogía en Educación General Básica) con 25 TFC cada una, seguidas muy de cerca por la línea de "Metodologías y evaluación en Educación Parvularia” (Pedagogía en Educación Parvularia), que tiene 24 TFC desarrollados. Hay ocho líneas de investigación que se encuentran $=/>$ dos TFC cada una, y tres de esas ocho líneas carecen de producción alguna de TFC.
Tabla 6.

Promedio de TFC por Lineas de investigación

\begin{tabular}{|l|c|c|c|}
\hline Carreras FECS & Líneas & TFC & Promedio \\
\hline P. Educación Física & 4 & 57 & 14.25 \\
\hline P. Música & $7^{*}(1)$ & 49 & 7 \\
\hline P. Biología & $6^{*}(1)$ & 21 & 3.5 \\
\hline P. Historia y Geografía & 3 & 23 & 7.67 \\
\hline P. Inglés & 4 & 33 & 8.25 \\
\hline $\begin{array}{l}\text { P. Lengua Castellana y } \\
\text { Comunicación }\end{array}$ & 4 & 26 & 6.5 \\
\hline Trabajo Social & $5^{*}(1)$ & 42 & 8.4 \\
\hline P. Educación Parvularia & 2 & 31 & 15.5 \\
\hline P. Matemática y Computación & 4 & 15 & 3.75 \\
\hline P. Educación General Básica & $4^{*}(1)$ & 79 & 19.75 \\
\hline P. Religión & 1 & 3 & 3 \\
\hline Totales & $\mathbf{4 4}$ & $\mathbf{3 7 9}$ & $\mathbf{1 1 . 6 1}$ \\
\hline
\end{tabular}

Las carreras que cuentan con mejor promedio de productividad en función de la relación líneas de investigación y TFC, son las carreras de Pedagogía en Educación General Básica (19.75), y Pedagogía en Educación Parvularia (15.5), seguidas muy de cerca por Pedagogía en Educación Física (14.25). Cuando el promedio de la FECS se halla en 11.61, estas tres carreras superan tal proporción.

En cuanto a la metodología desarrollada en los TFC (tabla 7), tenemos que, en términos generales, la metodología cuantitativa ha sido la de mayor abordaje $(64.91 \%)$, seguida de la metodología cualitativa $(29.02 \%)$ y la metodología mixta $(6.07 \%)$. La carrera de Pedagogía en Matemática y Computación es la que más ha empleado la metodología cuantitativa $(86.67 \%)$, seguido de Pedagogía en Educación 
Análisis bibliométrico de las tesis de pregrado en la Facultad de Educación y Ciencias Sociales de la Universidad Adventista de Chile, en el periodo 2008-2018

Física $(78.95 \%)$. No obstante, vale destacar que en la carrera de Pedagogía en Historia y Geografía, es la metodología cualitativa la que ha sido más desarrollada en los TFC (65.22 \%), al igual que sucede en la carrera de Trabajo Social (61.90\%). Pedagogía en Inglés vendría mixta en los TFC (21.21\%).

Tabla 7.

TFC según metodologías

\begin{tabular}{|c|c|c|c|c|c|c|c|c|}
\hline \multirow{2}{*}{ Carrera } & \multicolumn{2}{|c|}{ Cualitativa } & \multicolumn{2}{|c|}{ Cuantitativa } & \multicolumn{2}{|c|}{$\begin{array}{c}\text { Mixta/Propuesta } \\
\text { Didáctica }\end{array}$} & \multicolumn{2}{|c|}{ Totales } \\
\hline & $\mathbf{F}$ & $\%$ & $\mathbf{F}$ & $\%$ & $\mathbf{F}$ & $\%$ & $\mathbf{F}$ & $\%$ \\
\hline P. Educación Física & 9 & 15.79 & 45 & 78.95 & 3 & 5.26 & 57 & 100 \\
\hline P. Música & 18 & 36.79 & 30 & 61.22 & 1 & 2.05 & 49 & 100 \\
\hline P. Biología & 9 & 42.86 & 11 & 52.38 & 1 & 4.76 & 21 & 100 \\
\hline P. Historia y Geografía & 15 & 65.22 & 7 & 30.43 & 1 & 4.35 & 23 & 100 \\
\hline P. Inglés & 6 & 18.18 & 20 & 60.61 & 7 & 21.21 & 33 & 100 \\
\hline $\begin{array}{l}\text { P. Lengua Castellana y } \\
\text { Comunicación }\end{array}$ & 13 & 50 & 13 & 50 & 0 & 0 & 26 & 100 \\
\hline Trabajo Social & 26 & 61.90 & 15 & 35.71 & 1 & 2.39 & 42 & 100 \\
\hline P. Educación Parvularia & 3 & 9.68 & 24 & 77.42 & 4 & 12.90 & 31 & 100 \\
\hline $\begin{array}{l}\text { P. Matemática y } \\
\text { Computación }\end{array}$ & 2 & 13.33 & 13 & 86.67 & 0 & 0 & 15 & 100 \\
\hline $\begin{array}{l}\text { P. Educación General } \\
\text { Básica }\end{array}$ & 9 & 11.39 & 65 & 82.28 & 5 & 6.33 & 79 & 100 \\
\hline P. Religión & 0 & 0 & 3 & 100 & 0 & 0 & 3 & 100 \\
\hline Totales & 110 & 29.02 & 246 & 64.91 & 23 & 6.07 & 379 & 100 \\
\hline
\end{tabular}

En cuanto a la publicación de los TFC en revistas científicas indexadas (tabla 8), tenemos que el $2.90 \%$ de los mismos han sido publicados como artículos, teniendo por delante un $97.10 \%$ sin publicar, en el histórico de la producción de la FECS. Cuatro carreras han publicado TFC en el período en estudio, siendo estas, Pedagogía en Educación Física, con el 12.28 \% de sus trabajos; Pedagogía en Historia y Geografía, con el 8.70 \%; Pedagogía en Música, con el 2.04 de sus TFC, y cierra Pedagogía en Educación General Básica, con $1.32 \%$ de sus TFC publicados. 
Tabla 8.

Publicación de TFC en revistas cientificas indexadas

\begin{tabular}{|l|c|c|c|c|c|c|}
\hline \multirow{2}{*}{\multicolumn{1}{|c|}{ Carrera }} & \multicolumn{2}{c|}{ Publicados } & \multicolumn{2}{c|}{$\begin{array}{c}\text { No } \\
\text { publicados }\end{array}$} & \multicolumn{2}{c|}{ Totales } \\
\cline { 2 - 7 } & F & $\%$ & F & $\%$ & F & $\%$ \\
\hline P. Educación Física & 7 & 12.28 & 50 & 87.72 & 57 & 100 \\
\hline P. Música & 1 & 2.04 & 48 & 97.96 & 49 & 100 \\
\hline P. Biología & 0 & 0 & 21 & 100 & 21 & 100 \\
\hline P. Historia y Geografía & 2 & 8.70 & 21 & 91.30 & 23 & 100 \\
\hline P. Inglés & 0 & 0 & 33 & 100 & 33 & 100 \\
\hline $\begin{array}{l}\text { P. Lengua Castellana y } \\
\text { Comunicación }\end{array}$ & 0 & 0 & 26 & 100 & 26 & 100 \\
\hline Trabajo Social & 0 & 0 & 42 & 100 & 42 & 100 \\
\hline $\begin{array}{l}\text { P. Educación } \\
\text { Parvularia }\end{array}$ & 0 & 0 & 31 & 100 & 31 & 100 \\
\hline $\begin{array}{l}\text { P. Matemática y } \\
\text { Computación }\end{array}$ & 0 & 0 & 15 & 100 & 15 & 100 \\
\hline $\begin{array}{l}\text { P. Educación General } \\
\text { Básica }\end{array}$ & 1 & 1.32 & 78 & 98.68 & 79 & 100 \\
\hline P. Religión & 0 & 0 & 3 & 100 & 3 & 100 \\
\hline \multicolumn{1}{|c|}{ Totales } & 11 & 2.90 & 368 & 97.10 & 379 & 100 \\
\hline
\end{tabular}

Como podrá advertirse en la tabla 9, todos los TFC realizados en la FECS, en el período en cuestión, han sido desarrollados bajo la modalidad tesis, sin que se haya presentado alguno que haya adoptado las otras dos modalidades previstas en el reglamento de TFC que corresponden al tipo TFC proyecto $y$ TFC colaboración.

Tabla 9.

TFC según modalidad

\begin{tabular}{|l|c|c|}
\hline \multirow{2}{*}{\multicolumn{1}{|c|}{ Carrera }} & \multicolumn{2}{c|}{ Tesis } \\
\cline { 2 - 3 } & F & $\%$ \\
\hline P. Educación Física & 57 & 100 \\
\hline P. Música & 49 & 100 \\
\hline P. Biología & 21 & 100 \\
\hline P. Historia y Geografía & 23 & 100 \\
\hline P. Inglés & 33 & 100 \\
\hline P. Lengua Castellana y Comunicación & 26 & 100 \\
\hline Trabajo Social & 42 & 100 \\
\hline P. Educación Parvularia & 31 & 100 \\
\hline P. Matemática y Computación & 15 & 100 \\
\hline P. Educación General Básica & 79 & 100 \\
\hline P. Religión Totales & 3 & 100 \\
\hline \multicolumn{1}{|c|}{} & 379 & 100 \\
\hline
\end{tabular}

\section{Conclusiones}

Hacer investigación implica el desarrollo de habilidades específicas, más aún cuando la pesquisa se identifica con las inclinaciones temáticas y metodológicas de quien investiga, y cuando la investigación genera un impacto social, técnico, científico (Quijada, 2018). Si hablamos de la investigación en educación, se concibe su necesidad, a fin de transformar y potenciar las realidades en las que están inmersos quienes fungen como actores sociales determinantes. En tal sentido, la formación y desarrollo de habilidades investigativas en, y desde la práctica, forma parte del ideario del TFC en la FECS de la UnACh.

La investigación en educación ha sido desarrollada en la UnACh desde instancias básicas como los proyectos de investigación de los docentes (tributantes a fondos concursables), y los proyectos de investigación que surgen a partir de los TFC y que pueden cohesionarse sin problemas con proyectos en fondos concursables. Y es desde la instancia de los TFC desde la que se evidencia (según el interés particular de este trabajo) una producción que se ha mantenido regular en función de la matrícula existente en cada carrera tributante de la FECS. Esas investigaciones han permitido desarrollar líneas de investigación que tienen cierto vigor y respaldan su consolidación, si bien es cierto debe avanzarse aún más con la publicación y divulgación de los trabajos resultantes.

Asimismo, la declaración de las líneas de investigación de la FECS muestra que hay entre ellas algunas que no son consistentes y que no han tenido suficiente impulso para su desarrollo. En tal sentido, no sería justificable mantener líneas de investigación si no hay producción en las mismas. Una línea de investigación es un campo problémico (asociado al conocimiento) que se va delimitando, entonces tiene que ver con una perspectiva sistémica en la búsqueda y generación de conocimiento (Romero, en Agudelo, 2004). De esta forma, una línea tendría que emerger de las preocupaciones temáticas y problemas de conocimiento que se asocian de forma sistemática y organizada, y que se van contrastando con resultados de investigación. En 
concreto, no se crea una línea y después se agregan trabajos de investigación. La lógica indica lo contrario.

En ese sentido, la FECS necesita reorganizar líneas de investigación en función de las áreas de incidencia que atienden la formación universitaria, de aquellas que atienden al perfil de egreso de sus estudiantes en consonancia con el sello identitario de la institución, en articulación con las grandes áreas de investigación generadas por instancias superiores, como la Agencia Nacional de Investigación y Desarrollo (ANID) de Chile y el Ministerio de Educación (que atienden al campo laboral y al campo científico), y por una revisión continua de las tendencias internacionales en el campo de la investigación en educación.

$\mathrm{Al}$ considerar el tema de los docentes guía o asesores, se hace necesario destacar que en la $\mathrm{UnACh}$ se reconoce la tutoría como una actividad inherente a la formación universitaria en pregrado, y en tanto, se le atribuye vital importancia, dado su potencial para mejorar procesos y la calidad educativa (Sánchez, 2016). De esta manera se constata que la mayoría de los docentes en estas funciones cuenta con grado académico de Magíster y Doctorado. Este dato es importante dado que evidencia la fiabilidad en la competencia profesional en el proceso de guía de TFC. Tal moción se respalda en trabajos como el de Morillo (2010), para quien uno de los aspectos más importantes en la asesoría de tesis, aparte de la empatía, es precisamente la competencia profesional docente. A esto se suman Rosas et al. (2006, p. 1), para quienes "la influencia del tutor es muy relevante para determinar el éxito en la culminación del trabajo", asociado como está con la capacidad profesional para el ejercicio de la asesoría (Mogollón, 2006).

En relación con esto último, se encuentra el hecho de que varios docentes tienen un proceso de guiatura que se ha mantenido por años, y al mismo tiempo son docentes con altos credenciales profesionales en términos de grados académicos. Esto permite que el núcleo docente se nutra al mismo tiempo de la experiencia de los docentes con mayor trayectoria en la asesoría de TFC, lo que también se valida en otras investigaciones como Rosas et al. (2010) y Mogollón (2006).

Por otro lado, este estudio revela que las metodologías cuantitativas son las más empleadas en los TFC realizados en la FECS. De hecho, de las 11 carreras consideradas en el estudio, tan solo en dos de ellas (Pedagogía en Historia y Geografía y Trabajo Social) las metodologías cualitativas superan a las metodologías cuantitativas y a la mixtura, entre ambas opciones. No hay investigaciones que cuantifiquen la tendencia metodológica de otras facultades o escuelas de educación en los mismos términos en los que se ha realizado esta investigación. Jiménez (2004), quien realizó un análisis bibliométrico de las tesis de pregrado de estudiantes venezolanos en educación entre 1990 y 1999 en dos universidades: Universidad Central de Venezuela y Universidad Nacional Experimental Simón Rodríguez; clasificó la producción en función de las metodologías declaradas, sin detallar su caracterización como cualitativa, cuantitativa o mixta. Lo que encontró es que los tesistas declaran metodologías de investigación que en muchos casos no son coherentes con el desarollo de la investigación y superponen nombres para identificar la misma metodología. En sus palabras, dice: "se observan conceptos múltiples para explicar el mismo fenómeno y eso genera confusión” (p. 7).

Un segundo trabajo bibliométrico realizado en el interior de una escuela de educación es el de Moreira et al. (2019), en el que expone que si bien es cierto no se caracteriza la metodología empleada por los inves- 
tigadores en cada una de las tesis consideradas en el corpus del estudio, sí manifiestan que las imprecisiones metodológicas de las tesis no dan cuenta de los problemas de investigación que abordan (considerando casos de confusiones entre técnicas e instrumentos, confusión de objetivos, imprecisión en la declaración de instrumentos, entre otros). En el caso concreto de esta investigación, también ha podido evidenciarse que en algunos casos se han declarado metodologías que no son coherentes con el desarrollo de la investigación, procediéndose a rectificar el apartado metodológico para su correcta clasificación en el estudio.

Existen otras investigaciones que cuantifican la tendencia metodológica en carreras específicas [como lo es el caso de Lagos y Pérez-Gutiérrez (2016) para Educación Física], sin embargo, eso no permite la comparación con el presente trabajo porque cada carrera en particular tiene una tendencia marcada en función de los intereses de formación (declarado en las líneas de investigación que desarrollan, perfiles de egreso, programas de estudio en asignaturas tributantes a la investigación). No obstante, llama la atención la alta preponderancia de las metodologías cuantitativas en una facultad de Educación y de Ciencias Sociales. Eso podría estar determinado por la influencia del enfoque de investigación 'basado en evidencias científicas' (Serón, 2013) [proveniente del campo de la medicina (Campillo et al., 2019; Huicho et al., 2013; Martínez et al., 2014)], y eso en tanto hay quienes suponen que las metodologías cualitativas, o no se basan en evidencias científicas, o son muy débiles, o aún se encuentran en estado incipiente (Martínez et al., 2014).

Ahora bien, con respecto al tema de las publicaciones provenientes de TFC, tenemos que hasta el año 2017 en la UnACh no se había intentado publicar en revistas científicas indexadas trabajos provenientes de los TFC. Hasta ese momento, todos esos trabajos se venían desarrollando bajo la lógica tradicional de las tesis de licenciatura. No obstante, es a partir de 2018 cuando se toman decisiones en la FECS que tributan a la postulación de TFC en formato artículo, dando a los estudiantes la posibilidad de presentar el informe final con esas características.

Para el 2017, las publicaciones de la universidad en general (incluyendo todas las facultades, docentes y estudiantes) se encontraban por debajo del tope de 15 artículos publicados en revistas científicas indexadas al año (El Mercurio y Grupo de Estudios Avanzados Universitas, 2017). Si se toma en cuenta que en el país las universidades se clasifican en: universidades docentes, universidades docentes con proyección en investigación, universidades de investigación y doctorados en áreas selectivas, y universidades de investigación y doctorados, la UnACh (que ha mantenido entre 2017 y 2019 el primer lugar en el país en la categoría de Universidad Docente) se encontraba para 2017 con menos de 15 artículos publicados en revistas indexadas, y para la fecha, ninguno de esas publicaciones era producto de algún TFC. Desde entonces, se ha avanzado y una de las modalidades en la presentación de los TFC (no obligatoria) es precisamente la presentación en formato artículo. Tal medida ha potenciado la publicación de los 11 artículos provenientes de TFC en revistas indexadas entre 2018 y 2019 (véase tabla 8), sin contar con las publicaciones de los docentes investigadores de la universidad con poco más de 90 publicaciones. Para apoyar esa medida se fortalecen las instancias desde las que se pueda apoyar al estudiante en pos de la consecución de su TFC con instancias como, asesoría del tutor, apoyo de un asesor metodológico y un asesor estadístico, núcleos y grupos de investigación, jornadas de iniciación en la investigación, etc. 
Análisis bibliométrico de las tesis de pregrado en la Facultad de Educación y Ciencias Sociales de la Universidad Adventista de Chile, en el periodo 2008-2018

Figura 1.

Universidades de Chile, 2017

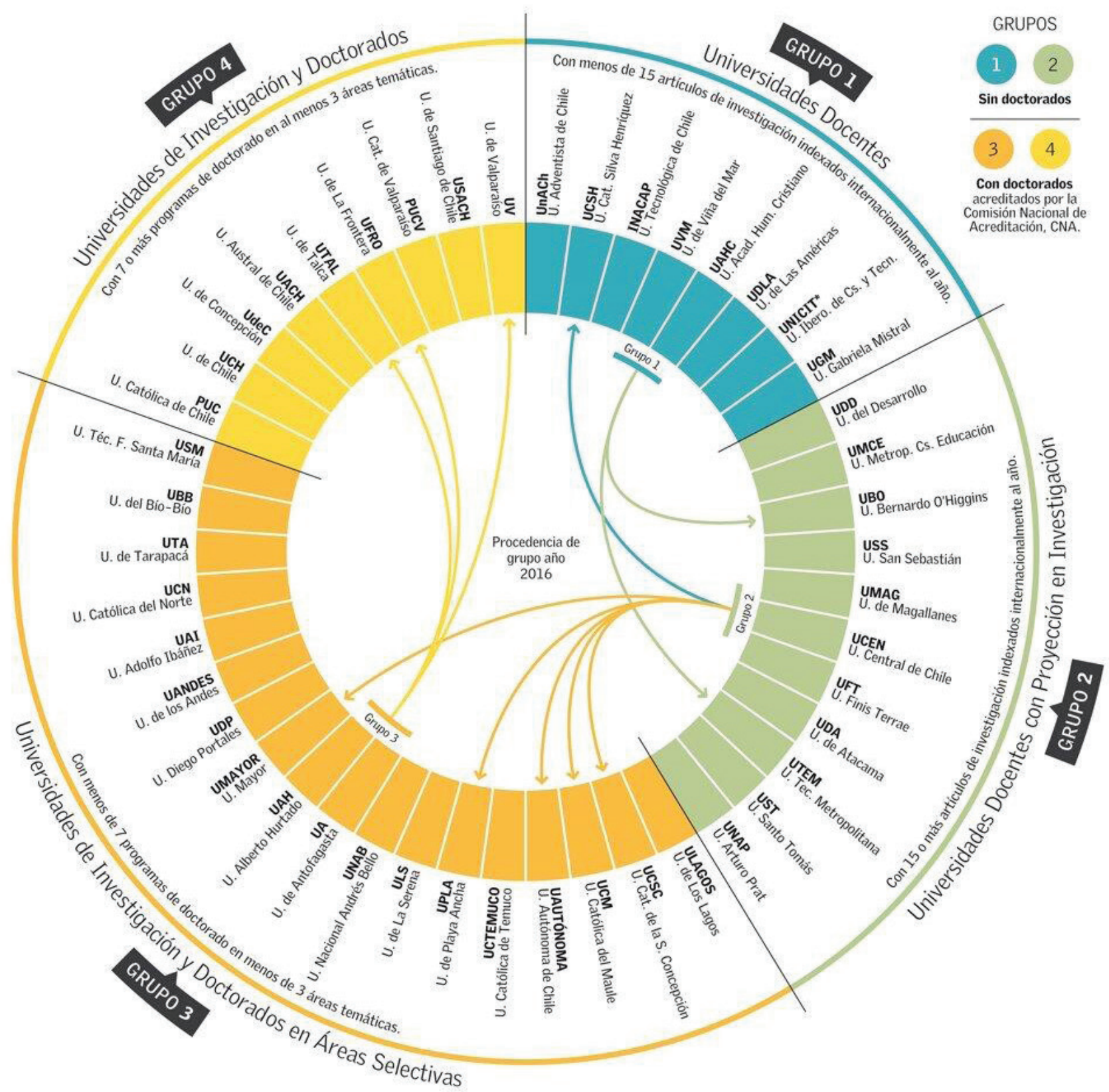

Nota: El Mercurio y Universitas Grupo de Estudios Avanzados en Educación Superior (2017).

Vale destacar que cuando se es estudiante de pregrado la publicación de trabajos en revistas científicas indexadas es bastante difícil (Ramos-Rodríguez y Sotomayor, 2008; Valle y Salvador, 2009). De hecho, hay revistas que rechazan trabajos cuando entre los autores hay estudiantes de pregrado (Huamaní et al.,
2008), y más aún, existen las revistas que solo publican a postdoctores, a doctores o a estudiantes de posgrados (Revista Educação \& Formação, 2020). Mucho de esto está relacionado también con el falso concepto de la poca calidad de esos trabajos (Jiménez-Peña et al., 2017). Pese a esto, también hay revistas científicas indexadas 
de muy buena calidad para estudiantes (Jiménez-Peña et al., 2017).

La escritura científica es una habilidad profesional de elevada complejidad y requiere el desarrollo de estrategias específicas para su consolidación. En tal sentido, la FECS está impulsando a estudiantes para los semilleros de investigación y aún desde las asignaturas de Métodos y Técnicas de Investigación I y II, y TFC para el desarrollo de habilidades asociadas a la redacción científica, entendiendo también que se trata de iniciar al estudiante en ese contexto, de ofrecer un agregado en la formación universitaria, porque la tesis o el TFC es una instancia para el desarrollo de habilidades básicas de investigación y no de publicación científica (Osada et al., 2010). Al iniciarse en el desarrollo de esta habilidad, el estudiante contará con mayores capacidades y podrá sentirse estimulado para investigar y publicar, contribuyendo con el crecimiento científico en su área de desempeño profesional.

Un rasgo de relevancia reside en el hecho de que la modalidad que se ha desarrollado en la FECS para presentar el TFC ha sido la tesis, de forma exclusiva. Si bien es cierto que se ha avanzado en la presentación del TFC en formato artículo, sería válido incorporar, a la modalidad tesis, otras modalidades de presentación de trabajo final, tal y como se expresa en el Reglamento del Trabajo Final de Carrera para los Estudios de Pregrado (2017). Y esto, habida cuenta que también permite el desarrollo de habilidades investigativas, e incluso, puede anticipar la formación según la modalidad, como lo es el caso del proyecto o de la colaboración.

Más allá de caracterizar y cuantificar la producción científica de la FECS en términos de los TFC (20082018), este trabajo permite visualizar las tendencias de investigación en educación, tal y como ha venido siendo estudiado no solo desde el campo de la bibliometría, sino también desde trabajo de Piaget et al. [1973 (publicado originalmente en 1970)], que permite evidenciar y conocer la dinámica de investigación hacia líneas específicas y subáreas temáticas, enfoques (cualitativos, cuantitativos, mixtos) que, de una forma u otra tienen un impacto en la formación inicial docente.
El trabajo formativo que se realiza desde asignaturas que tributan a la adquisición y desarrollo de habilidades investigativas es fundamental. Se hace referencia explícita a asignaturas como Métodos y Técnicas de Investigación I y II, Formulación de Proyectos y TFC. Sin embargo, se constata que para que se logre tener mayor adherencia en este proceso, se hace preciso abordar habilidades básicas desde la transversalidad del currículo, esto es, todas las asignaturas pueden tributar al desarrollo de estas habilidades.

Todas las carreras de la FECS que en el período en estudio (2008-2018) han tenido cohortes en proceso de TFC, han mostrado la producción en tales términos. Como se ha mostrado, las carreras de Pedagogía en Educación General Básica y Pedagogía en Educación Física son las más productivas, y esto está relacionado directamente con la matrícula en ambas carreras, por el mismo trabajo que ha sido desarrollado a lo interno de las carreras con el equipo de docentes. Si asociamos la publicación de los TFC, tendríamos que Pedagogía en Educación Física se manifiesta como la de mayor productividad científica en la FECS. Esta, de forma general, se ha preocupado y ha logrado contar con un alto número de docentes asesores de TFC con estudios de posgrado (magíster y doctorado), lo que también permite consolidar habilidades de investigación en los estudiantes en fase de desarrollo de TFC, al estar acompañados de docentes capacitados en investigación.

Por otro lado, hay variedad de líneas de investigación en cada una de las carreras para que los docentes y los estudiantes desarrollen sus respectivos proyectos y TFC. No obstante, la Dirección de Investigación de la universidad requiere que una línea se mantenga vigente cuando, en orden jerárquico, cumple con alguno de los siguientes requerimientos: 1- tiene y mantiene productividad comprobada en la publicación de artículos científicas en revistas indexadas de alto impacto, y/o libros científico-académicos arbitrados; 2 - tiene proyectos de investigación en fondos internos y/o externos; 3- tiene por lo menos dos TFC desarrollados en un área temática. En este sentido, hay líneas de investigación declaradas que no han 
alcanzado los mínimos niveles de productividad, por lo cual se requeriría una redefinición de líneas y eliminación de aquellas en las que no hay investigación, dado que la investigación es la que va creando una línea de investigación y no viceversa.

\section{Referencias}

Agramonte, E. D., Díaz, A. A., Hernández S. A., Hernández, J. L., Salomón, Y. P. y Miranda, M. B. (2018). La investigación sobre Tecnologías de la Información y las Comunicaciones en la Facultad de Comunicación de la Universidad de La Habana: estudio bibliométrico de las tesis de pregrado y posgrado (2006-2016). Ponencia presentada en el Congreso Internacional de Información INFO'2018, 5 al 9 de marzo, La Habana, Cuba. https://cutt.ly/8jsJflp

Agudelo, N. (2004). Las líneas de investigación y la formación de investigadores: una mirada desde la administración y sus procesos formativos. Revista ieRed: Revista Electrónica de la Red de Investigación Educativa, 1(1), 1-11. https://cutt. ly/0tZcJUy

Aleixandre-Benavent, R., Gonzáles de Dios, J., Castelló Cogollos, L., Navarro Molina, C., Alonso-Arroyo, A., Vidal-Infer, A. y Lucas-Domínguez, R. (2017). Bibliometría e indicadores de actividad científica (1). La evaluación de la investigación y de la actividad científica en pediatría a través de la bibliometría. Acta Pediátrica Española, 75(1-2), 18-25. https://cutt.ly/ktZjK5P

Alonso Jiménez, V. (2008). Guía metodológica para elaborar proyectos de investigación en Ciencias Políticas y Administración Pública. Espacios Públicos, 11(23), 226-247. https://cutt.ly/ HjsJK8Y

Arellano-Sacramento, C., Hermoza-Moquillaza, R. V., Elías-Podestá, M. y Ramírez-Julca, M. (2017). Actitud hacia la investigación de estudiantes universitarios en Lima, Perú. FEM: Revista de la Fundación Educación Médica, 20(4), 191-19. https://cutt.ly/WjsJMgv
Arias Monge, N. y Navarro Camacho, M. (2017). Epistemología, Ciencia y Educación Científica: premisas, cuestionamientos y reflexiones para pensar la cultura científica. Revista Electrónica Actualidades Investigativas en Educación, 17(3), 1-20. https://cutt.ly/UjsJ5uL

Arnoux, E. (2008). Escritura y producción de conocimiento en las carreras de posgrado. Buenos Aires: Santiago-Arcos.

Barra, A. M. (2019). La importancia de la productividad científica en la acreditación institucional de universidades chilenas. Formación Universitaria, 12(3), 101-110. https://cutt.ly/tjsKu6D

Bird, S. (2014). Socially Responsible Science is More than "Good Science". Journal of Microbiology \& Biology Education, 15(2), 169-172. https:// cutt.ly/GjsKUu6

Campillo Labrandero, M., Pompa Mansilla, M., Hernández Carrillo, F. y Sánchez Mendiola, M. (2019). Una experiencia de formación: la Educación basada en evidencias. Revista Digital Universitaria, 20(6), 1-10. https://cutt.ly/MjsKktw

Castro-Rodríguez, Y. (2018). Indicadores bibliométricos de las tesis sustentadas por estudiantes de Odontología, Perú. Edumecentro, 10(4), 1-19.

Chuaqui, B. (2002). Acerca de la historia de las universidades. Revista Chilena de Pediatría, 73(6), 563-565.

Cortés Vargas, D. (2007). Medir la producción científica de los investigadores universitarios: La bibliometría y sus límites. Revista de Educación Superior, XXXVI 2(142), 43-6.

Coto Jiménez, M. (2020). Análisis bibliométrico de los proyectos de graduación de ingeniería eléctrica de la Universidad de Costa Rica 1999-2018. E-Ciencias de la Información, 10(1), 3-22. http://dx.doi.org/10.15517/eci.v10i1.39883

El Mercurio y Grupo de Estudios Avanzados Universitas (2017). Ranking de calidad de las Universidades Chilenas. https://cutt.ly/7tZcS1s

Ganga, F., Ortiz-Salgado, R. y Valassina, F. (2019). Estrategias tendientes a incrementar las publicaciones académicas. Caso Universidad del Bío-Bío de Chile. Opción, 38(89-2), 435-469. https:// cutt.ly/mjsK7jf 
Garrido, M. R. (2011). Fundamentos del análisis documental, en: J. López Yepes y M. d. R. Osuna Alarcón (Eds.), Manual de Ciencias de la Información y Documentación (pp. 279-291). Madrid: Pirámide.

Hederich, C., Martínez Bernal, J. y Rincón Camacho, L. (2014). Hacia una educación basada en la evidencia. Revista Colombiana de Educación, 66, $19-54$.

Hirsch Adler, A. (2020). Profesores universitarios y responsabilidad social de la ciencia. Ciencia $y$ Educación, 4(2), 103-114. https://doi.org/10. 22206/cyed.2020.v4i2.pp103-114

Huamaní, Ch., Chávez-Solís, P. y Mayta-Tristán, P. (2008). Aporte estudiantil en la publicación de artículos científicos en revistas médicas indizadas en SciELO-Perú, 1997-2005. Anales de la Facultad de Medicina, 69(1), 42-45. https:// cutt.ly/RtZcA8T

Huicho, L., Carreazo, N. Y. y González, C. (2013). La Medicina Basada en la Evidencia: ¿mejoró la medicina que practicamos y enseñamos? Anales de la Facultad de Medicina, 74(3), 231-235. https:/cutt.ly/6jsLrS2

Ibarra-López, P. (2017). La titulación por tesis en México. El problema de su conceptualización. Revista Dilemas Contemporáneos: Educación, politica y valores, IV(3), 1-22. https://cutt.ly/mtTWQwp

International Organization for Standardization (2010). UNE-ISO 690: 2010. Información y documentación. Directrices para la redacción de referencias bibliográficas y de citas de recursos de información. Madrid: AENOR.

Jiménez, E. (2004). Análisis bibliométrico de tesis de pregrado de estudiantes venezolanos en el área de educación: 1990-1999. Revista Iberoamericana de Educación, 1-15. https://cutt.ly/ $\mathrm{XjsLoCZ}$

Jiménez-Peña, D., Serrano, F. T. y Pulido Medina, C. (2017). Publicación en revistas científicas estudiantiles. ¿La respuesta a la problemática de dónde publicar en el pregrado? Revista Médica de Chile, 145, 816-820. https://cutt.ly/ $\mathrm{FtZcOGD}$
Lagos, R. I. y Pérez-Gutiérrez, M. (2016). Análisis bibliométrico de las tesis de pregrado: el caso de Pedagogía en Educación Física de la Universidad Autónoma de Chile (2007-2012). Revista Iberoamericana de Educación, 70(1), 181-200. https://cutt.ly/fjsLsXk

Madrid Martín, M. J., Jiménez-Fanjul, N., León-Mantero, C. y Maz-Machado, A. (2017). Revistas brasileñas de Educación en SCOPUS: un análisis bibliométrico. Biblios, 67, 30-41. http:// dx.doi.org/10.5195/biblios.2017.344

Maggiaroni, J., Cloquell, D., Izarra, E. y Bastardo, K. (2019). Estudio bibliométrico de los trabajos especiales de grado de la Facultad de Odontología de la Universidad de Los Andes 2005-2017. Revista Venezolana de Investigación Odontológica de la IADR, 7(1), 21-4.

Michán, L. y Muñoz-Velasco, L. (2013). Cienciometría para ciencias médicas: definiciones, aplicaciones y perspectivas. Investigación en educación médica, 2(6), 100-106. https://cutt.ly/djsLjFj

Millán, J. D., Polanco, F., Ossa, J. C., Suelí, J. y Cudina, J. N. (2017). La cienciometría, su método y su filosofía: Reflexiones epistémicas de sus alcances en el siglo XXI. Revista Cientifica Guillermo de Ockham, 15(2), 17-27. http://dx.doi.org/10. 21500/22563202.3492

Mogollón de González, A. (2006). Éxito del tutor universitario. Revista Ciencias de la Educación, 6(1), 109-122. https://cutt.ly/ujsLTio

Moncada, J. S. (2008). La universidad: un acercamiento histórico-filosófico. Ideas y valores, (137), 131-148. https://cutt.ly/djsLjFj

Moreira Ramírez, L. V., Periguachi Nazareno, P. X. y Cano De la Cruz, Y. (2019). Análisis bibliométrico de los trabajos de titulación de la Escuela de Ciencias de la Educación en la PUCE SD. Revista Magazine de las Ciencias, 4(1), 81-91. https://cutt.ly/WjsLcJr

Morillo Moreno, M. C. (2009). Labor del tutor y asesor del trabajo de investigación. Experiencias e incentivos. Educere, 13(47), 919-930. https:// cutt.ly/5tTVILA 
Muñoz Cadena, O. J. y Suárez, M. A. (2018). Análisis bibliométrico de los trabajos de grado de la Facultad de Psicología de la Universidad San Buenaventura Bogotá (2002-2012) (Trabajo de grado). Universidad San Buenaventura, Bogotá, Colombia. https://cutt.ly/MjsLIBF

Osada, J., Ruiz-Grosso, P. y Ramos, M. (2010). Estudiantes de pregrado: el futuro de la investigación. Revista Peruana de Medicina Experimental y Salud Pública, 27(2), 305-306. https://cutt. ly/EjsLUcv

Peralta González, M. J., Frías Guzmá, M. y Chaviano, O. G. (2015). Criterios, clasificaciones y tendencias de los indicadores bibliométricos en la evaluación de la ciencia. Revista Cubana de Información en Ciencias de la Salud, 26(3), 290-309.

Piaget, J., Lazarsfeld, P. F., McKenzie, W. J. M. (1973). Tendencias de la investigación en ciencias sociales. [Versión PDF]. Alianza/UNESCO. https://cutt. ly/ntZcUZP

Piedra S. y Martínez R., A. (2007). Producción científica. Ciencias de la información, 38(3), pp. 33-38.

Quijada Lovatón, K. Y. (2018). Proactividad, pasión por el conocimiento y resistencia, cualidades para desarrollar la excelencia en la investigación. Sinéctica, (51), 1-19. https://cutt.ly/IjsLLXP

Ramos-Rodríguez, M. y Sotomayor R. (2008). Realizar o no una tesis: Razones de estudiantes de medicina de una universidad pública y factores asociados. Revista Peruana de Medicina Experimental y Salud Pública, 25(3), 322-324. https://cutt.ly/yjsLJqC

Reinders, J. J., Kropmans, T. J. y Cohen-Schotanus, J. (2005). Extracurricular research experience of medical students and their scientific output after graduation. Medical Education, 39(2), 237. https://cutt.ly/TjsL19Z

Revista Educacao \& Formacao (2020). Informação para Autores. https://cutt.ly/SjsL3iS

Reyes, A. y Moraga, R. (2020). Criterios de selección de una revista científica para postular un artículo: breve guía para no 'quemar' un paper. Sophia, 16(1), 93-109.
Rosas, A. K., Flores, D. y Valarino, E. (2006). Rol del tutor de tesis: competencias, condiciones personales y funciones. Investigación y Postgrado, 21(1). https://cutt.ly/3jsL6Bm

San Martín H., F. y Pacheco, J. (2008). Análisis bibliométrico de las tesis de pregrado de la Facultad de Medicina Veterinaria de la Universidad Nacional Mayor de San Marcos en el período 2001-2006. Revista de Investigaciones Veterinarias del Perú, 19(1), 82-92.

Sánchez Encalada, M. L. (2016). Formación de tutores como estrategia para mejorar el rendimiento académico de los estudiantes universitarios. Integración Académica de Psicologia, 4(12), 56-68. https://cutt.ly/UtTBgE7

Sánchez, R. (2014). Enseñar a investigar. Una didáctica nueva de la investigación en ciencias sociales $y$ humanas. Universidad Nacional Autónoma de México.

Serón S., P. [CIGES UFRO]. (2013, octubre 8). Aspectos metodológicos de las revisiones sistemáticas. [Archivo de video]. https://cutt.ly/ LjsZhwn

Tomás-Górriz, V. y Tomás-Casterá, V. (2018). La Bibliometría en la evaluación de la actividad científica. Hospital a Domicilio, 2(4),145-163.

Universidad Adventista de Chile (2018). Informe de autoevaluación institucional 2018. Chillán: Universidad Adventista de Chile.

Universidad Adventista de Chile (2017). Reglamento del Trabajo Final de Carrera para los Estudios de Pregrado. Chillán: Universidad de Chile. https:// cutt.ly $/ 7 \mathrm{tZzXQf}$

Valle, R. y Salvador, E. (2009). Análisis bibliométrico de las tesis de pregrado de la Facultad de Medicina de la Universidad Nacional Mayor de San Marcos. Anales de la Facultad de Medicina, 70(1), 11-18. https://cutt.ly/XjsZBIo

Velasco, B., Eiros, J. M., Pinilla, J. M. y San Román, J. A. (2012). La utilización de los indicadores bibliométricos para evaluar la actividad investigadora. Aula Abierta, 40(2), 75-84. 
Alixon Reyes Rodríguez, Julia Labra, Bertha Méndez, Lorena Gutiérrez-Jeldres, Leonel Federico-Tuccelli y Fanny Vezzoli-Lara

\section{Agradecimientos}

A los directores de las diferentes carreras de la FECS, a la Coordinación de TFC y a la Biblioteca Mariano Renedo Lucero, por el aporte de información en relación con líneas de investigación y TFC. 